\title{
Effects of DOF Separation on Elastic Devices for the Navigation in 3D Virtual Environments with Force Feedback
}

\author{
Géry Casiez, Christophe Chaillou \\ LIFL (UMR CNRS 8022) \& INRIA Futurs \\ Université des Sciences et Technologies de Lille, \\ 59655 Villeneuve d'Ascq, France \\ \{gery.casiez, christophe.chaillou\}@lifl.fr
}

\begin{abstract}
We investigated the use of a new haptic device called DigiHaptic in a 3D navigation task. Unlike other devices intended to interact in $3 D$ with one end-effector, the DigiHaptic has three levers that the user may handle simultaneously or not in elastic mode to rate control objects. We compared it to the SpaceMouse - another elastic device - to evaluate the influence that degrees of freedom (DOF) separation and force feedback have in terms of accuracy (errors) and speed (time) in a $3 D$ navigation task. We found that users performed the task with the same amount of time on both devices but that DOF separation increases the control accuracy of the camera.
\end{abstract}

\section{Introduction}

A navigation task in a $3 \mathrm{D}$ virtual environment comprises two subtasks: the wayfinding and the control of the user point of view [1]. The first is a cognitive process which leads the user to control the point of view of the camera in the scene. The control of the point of view is closely related with the device used. The device can be isotonic, elastic or isometric and the point of view can be controlled using position or rate control. Zhai showed that isotonic devices are better in position control tasks and both elastic and isometric devices are better in rate control tasks [6].

The difficulty to control the point of view of the camera increases with the number of DOF of the device. As a result, metaphors proposing DOF separation on devices were proposed [7] to improve user efficiency.

As it appears that DOF separation seems to increase the user ability to control the camera, we have developed a new three DOF haptic device, called DigiHaptic, proposing the separation of the degrees of freedom [3]. The device comprises three levers associated with the thumb, forefinger and ring finger and it can be used in isotonic mode for position control or elastic mode for rate control by simulating springs on each lever. In each mode, forces can be rendered on each finger.

In this paper, we investigate the use of the DigiHaptic in elastic mode for the navigation in $3 \mathrm{D}$ virtual environments to analyse the effect of DOF separation on elastic devices for the navigation in $3 \mathrm{D}$ virtual environments. With this aim in view, we will compare the separated DOF of the DigiHaptic to the integrated DOF of the SpaceMouse.

\section{Experiment}

The objective of this study is to investigate human ability to control three separated elastic DOF in a 3D navigation task. This includes the time and accuracy required to perform the task. We also evaluate the effect of force feedback on performances when a user touches the sides of the tunnel.

\subsection{Apparatus}

We used a DigiHaptic and a SpaceMouse Classic as input devices. For the DigiHaptic, fixed spring stiffnesses were set on each lever and remained the same for all subjects. Both elastic devices were operated in rate control mode. Additional force feedback was possible on the DigiHaptic when the user hit the sides of the tunnel. To ensure a fair comparison between the two devices, we tuned the sensitivities for the two devices with two experimented users on each of them. We have chosen the average sensitivity for each device which leads to the lowest completion time.

The experiment was done in full-screen mode on a 21 inch monitor running at a resolution of 1024 by 768 pixels. 


\subsection{Task}

The task consisted in navigating a 3D tunnel (figure 1) by controlling three degrees of freedom of the camera. We designed the tunnel using a BSP file editor. We then wrote a custom application to load BSP files, operate the devices, check collisions and calculate force feedback.
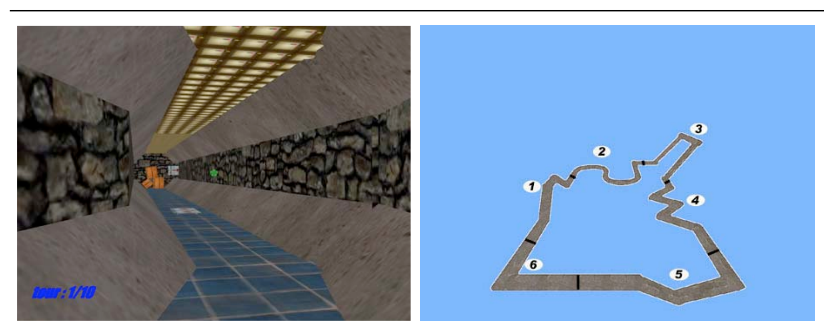

Figure 1. Screenshot inside the tunnel (left) and a global view of the whole 3D tunnel (right).

We used the "flying vehicle control" metaphor [5] with rate control to navigate in the $3 \mathrm{D}$ tunnel as it appears to be the best metaphor to control the camera using rate control.

For the DigiHaptic, the thumb controls the rotation of the camera around the vertical axis (yaw), the ring finger controls the rotation of the camera around the horizontal axis (pitch) and the index controls the forward/backward movement of the camera. For the SpaceMouse, the same degrees of freedom of the camera are controlled (figure 2). Each time, there is an isomorphism between the DOF controlled on the device and the DOF controlled on the camera, which makes the use of each DOF intuitive. We could have used a three DOF joystick (with yaw control on the stick) instead of using a SpaceMouse. Unfortunately it would not have made it possible to get the isomorphisms due to the left - right movement of the stick.

The tunnel is divided in six segments of different shapes (figure 1). The user starts at the beginning of the first segment and navigate the tunnel clockwise.

For the ground, the sides and the ceiling, different textures are used as visuals clues to provide references to the user. During the progression in the tunnel, arrows on the ground and on the walls are always visible to indicate the right direction to follow. Boxes with wood texture are placed at each corner to help the user to anticipate the turning. A green point located at the screen center helps the user to position the camera. At last, the number of laps realized appears in blue at the left bottom of the screen.

In case of collision with the tunnel, the camera is slowed or stopped proportionally to the angle between the camera and the wall. In the case of the use of the DigiHaptic with force feedback, the collision forces are only sent to the fore-

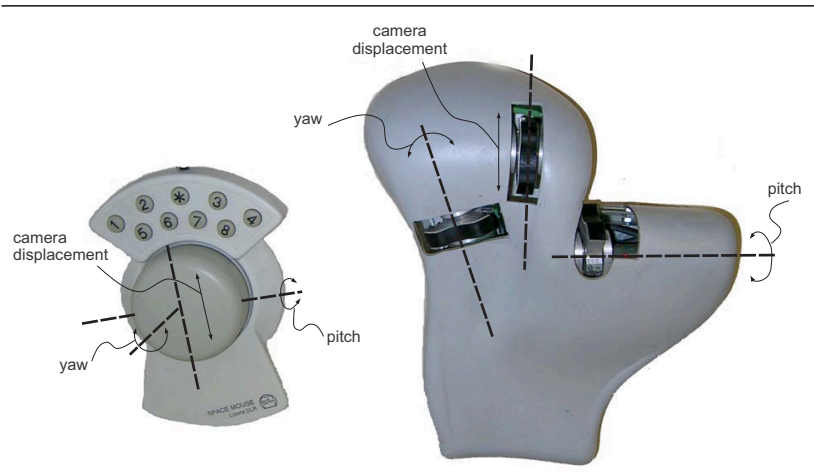

Figure 2. Degrees of freedom of the camera controlled with the DigiHaptic and the SpaceMouse.

finger. As it controls the displacement of the camera, it is natural to send the collisions forces to this finger to reduce the camera speed. We could also have sent force feedback on the thumb and ring finger to simulate torque when the camera collides with a wall. Nevertheless, we did not activate it because simulation of torque would have helped the user to escape from the wall and would not have permitted to fairly evaluate the influence of force feedback.

\subsection{Participants}

A group of 30 subjects volunteers, aged 19-38 years old, participated in the experiment. All subjects (26 were right-handed and 4 left-handed) used their right hand on each device. The left-handed subjects, were in the habit of using their right hand to work with the computer mouse, and had no special difficulty compared to right-handed subjects in using the DigiHaptic or the SpaceMouse. All subjects were used in playing 3D video games. Participants were separated in two groups. The first group tested the SpaceMouse (SM) and the DigiHaptic without force feedback (DH) (group 1) and the second group tested the SpaceMouse (SM) and the DigiHaptic with force feedback (DHFF) (group 2). To counterbalance the effect of learning, half of the participants of each group started with the DigiHaptic and the other one with the SpaceMouse. None of the subjects had prior experience with the task involved or the devices used.

\subsection{Procedure}

Subjects sat in front of the screen at an average distance of $70 \mathrm{~cm}$. Tests started after a training session during which the user got used in using the device in the environment. Subjects could train as long as they wanted until they felt ready to start. On average, each subject realized five laps during the training session. 
During the test session, participants were instructed to realize the task as fast as possible while minimizing the number of contacts with the tunnel. A contact was recorded each time the camera hit a side of the tunnel.

The session ended with a questionnaire. Then the participants started with the second device.

The time, the position of the camera and the number of contacts were recorded each $30 \mathrm{~ms}$ on average.

\section{Results}

\subsection{Time}

The mean time to perform a lap is very close for the two devices (figure 3 ) and tends to decrease as the number of laps increases. For each group, the differences between the two devices are not significant.

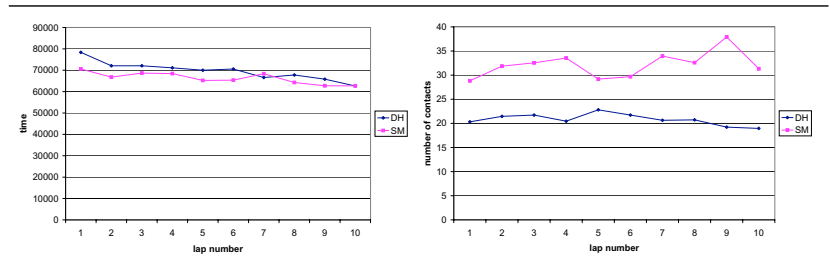

Figure 3. Mean time per lap (in ms), for the DigiHaptic and the SpaceMouse (groups 1 and 2 gathered) (left) and mean contact number per lap, for the DigiHaptic and the SpaceMouse (groups 1 and 2 gathered) (right).

\subsection{Number of contacts}

The mean contact number per lap is always greater for the SpaceMouse (figure 3) and this difference is significant for each group $(p<0.04)$.

No significant effect was found for the difference between the number of contacts for the DigiHaptic with force feedback (group 2) and the DigiHaptic without force feedback (group 1).

\subsection{Contact duration}

For the subjects of the first group, the difference between the two devices is not significant. In return the difference is significant for the subjects of the second group $(p<0.03)$. We expected this result because force feedback tends to push back the camera from the wall.

\subsection{Results per segments}

We computed the results for each segment defined in figure 1 to analyse the influence of the shape of the tunnel. Concerning the time, there is no significant difference between the two devices.

In return, the differences are significant for the number of contacts per segment. They are significant for the two groups for the segments 2 and 4 : the segment with the two arcs of circle and the one with the zigzags, which are especially difficult.

\subsection{Number of DOF used}

We also computed the number of DOF simultaneously used on the DigiHaptic and the SpaceMoue. A DOF is supposed to be used if it is modified between two steps of time. This is an application of an article by Jacob et al. where a similar analysis is performed on isotonic devices [4].

From the data collected each $30 \mathrm{~ms}$ from the whole subjects of the two groups, we computed the number of DOF modified on each step of time. The critical point of this method is to determine the value above which a modification is considered. If this value is too low, unwanted movements can be considered as intentional movements of the user. At opposite a large value would neglect intentional movement of the user. We empirically considered a DOF was modified if the angle variation was more important than $0,15^{\circ}$ for both devices. A variation of this value from $50 \%$ to $200 \%$ does not globally change the results.

The data were submitted to a one-way analysis of variance (ANOVA) with the number of modified as the independent factor at three levels (one, two or three DOF modified). This analysis showed a significant effect of the number of DOF modified for the DigiHaptic $\left(F_{2,87}=245.7 ; p<\right.$ $\left.0.0001 ; R^{2}=0.85\right)$. Users try to minimize the number of DOF used on it (figure 4).

We can notice that most of the time, no DOF is modified on the SpaceMouse. Here no significant effect was found when DOF are modified.

\section{Discussion and conclusion}

In the comparison study between the DigiHaptic and the SpaceMouse, we noticed that the mean time per lap are equivalent for the two devices and that the time differences between the two devices were not significant. The sensitivities tuning is not responsible for that fact as they are judged similar for the two devices. So we can not conclude to the superiority of one device from the task completion time point of view. From the questionnaire qualitative results, users indicated however that they expected to go faster with the DigiHaptic after a longer training period. 


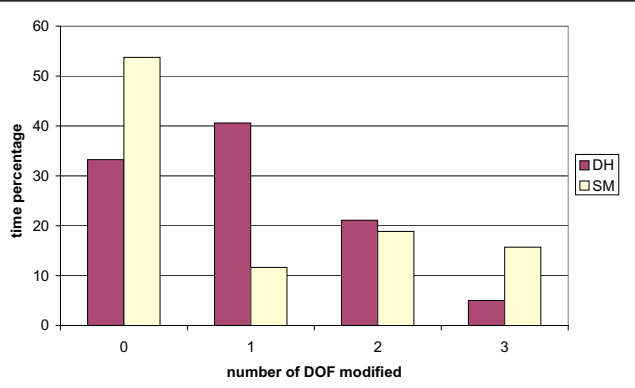

Figure 4. Percentage of time against the number of DOF modified.

From the accuracy point of view, we saw that the DigiHaptic significantly reduced the number of contacts with the tunnel sides. The DigiHaptic allows a better control of the camera, in particular in the segments requiring the use of only two DOF simultaneously which are judged particulary difficult (segments 2 and 4). In these segments, where the use of the thumb and forefinger are only required, the adjustment of the trajectory is permanent. For the other segments, the results are less significant because part of the users tend to confound the use of the forefinger and ring finger and because the position of the ring finger is less ergonomic. Thus, the isomorphism between the degrees of freedom of the camera for the index and ring finger is not so easy for everybody. From the ergonomic issues stated by the users we developed a new version of the device which is not presented in this paper. The worst results for the SpaceMouse can be partly explained by the fact that part of the users find the device particulary tiring. We also think that the ability to control one degree of freedom at a time leads to a more precise control of the camera. Indeed users minimize the number of DOF modified on the DigiHaptic.

Due to the difference in the number of contacts between the two devices, we could have expected to reduce the mean lap time with the DigiHaptic. This can be explained by the fact that users navigate faster with the SpaceMouse between two contacts and that users navigate slower with the DigiHaptic because it makes it possible to have more control on the speed.

As we expected the force feedback reduces the contact duration. Even if it is not considered as essential, it is appreciated by the users. Here, force feedback can be seen as an information such as sound would be.

For the muscular fatigue, the SpaceMouse is more tiring than the DigiHaptic. Indeed, it requires more muscular contractions to control the end-effector which has a low range of movement compared to the DigiHaptic.

The results of this study allow us to think that the DigiHaptic, preferred by the users, requires a longer training period to take in hand but leads to better results than with the
SpaceMouse. We think this device makes sense as a not expansive three DOF elastic force feedback device for navigation applications.

The DigiHaptic was previously evaluated and compared to the SpaceMouse in a 3D steering task experiment were it showed to double the task completion time while increasing the accuracy [2]. The difference between the results of the two experiments can be explained by the fact that the navigation task is a more separated task [4] compared to the steering task. Jacob showed that integrated isotonic devices are more adapted to integrated task, and that separated isotonic devices were more adapted to separated task [4]. Here, we obtain a similar result for elastic devices.

As future work, we want to evaluate the force feedback on three degrees of freedom to compare with the force feedback on one degree of freedom.

\section{Acknowledgements}

This work has been carried out within the framework of the INRIA Alcove project and is supported by the IRCICA (Institut de Recherche sur les Composants logiciels et matériels pour l'Information et la Communication Avancée). This work would not have been possible without the contribution of Sylvain Henot for the development of the navigation application.

\section{References}

[1] D. Bowman, D. Koller, and L. Hodges. A Methodology for the Evaluation of Travel Techniques for Immersive Virtual. Virtual Reality: Research, Development and Applications, 3(2):120-131, 1998.

[2] G. Casiez, P. Plénacoste, and C. Chaillou. Does DOF Separation on Elastic Devices Improve User 3D Steering Task Performance? In L. . S.-V. B. Heidelberg, editor, Asia-Pacific Conference on Computer-Human Interaction (APCHI'04), pages 70 - 80, June 29 - July 022004.

[3] G. Casiez, P. Plénacoste, C. Chaillou, and B. Semail. The DigiHaptic, a New Three Degrees of Freedom Multi-finger Haptic Device. In Proceedings of Virtual Reality International Conference, pages 35-39, May 2003.

[4] R. Jacob, L. Silbert, C. Mcfarlane, and M. Mullen. Integrality and Separability of Input Devices. ACM Transactions on Computer-Human Interaction, 1(1):3-26, March 1994.

[5] C. Ware and S. Osborne. Exploration and virtual camera control in virtual three dimensional environments. In Proc. of ACM Symposium on Interactive 3D Graphics, pages 175-183, 1990.

[6] S. Zhai. Human Performance in Six Degree of Freedom Input Control. PhD thesis, University of Toronto, 1995.

[7] S. Zhai, E. Kandogan, B. Smith, and T. Selker. In Search of the "Magic Carpet", Design and Experimentation of a 3D Navigation Interface. Journal of Visual Languages and Computing, 10(1):3 - 17, 1999. 